Research Article

\title{
The Psychological Benefits of COVID-19 Vaccination
}

\author{
My Nguyen (iD) \\ Faculty of Economics and Public Management, Ho Chi Minh City Open University, Ho Chi Minh City, Vietnam
}

Correspondence should be addressed to My Nguyen; my.t.nguyen93@gmail.com

Received 3 July 2021; Accepted 12 October 2021; Published 3 November 2021

Academic Editor: Carol J. Burns

Copyright (C) 2021 My Nguyen. This is an open access article distributed under the Creative Commons Attribution License, which permits unrestricted use, distribution, and reproduction in any medium, provided the original work is properly cited.

\begin{abstract}
This study evaluates the extent to which COVID-19 vaccination affects population mental health. Exploiting the within-state and within-survey week variation in the number fully vaccinated per 10 million people, I uncover the favorable effects of vaccination on individuals' psychological well-being. Particularly, a 100\% increase in the number fully vaccinated per 10 million people makes individuals $0.24,0.23,0.12$, and 0.14 percentage points less likely to experience anxiety, worry, displeasure, and depression on a daily basis, respectively. The probability of having at least one of the four symptoms (anxiety, worry, displeasure, and depression) every day also reduces by 0.27 percentage points. The study calls for the expansion of vaccine coverage, especially for disproportionately affected communities.
\end{abstract}

\section{Introduction}

The outbreak of the coronavirus disease 2019 (COVID-19) in China in late 2019 has posed major public health and governance challenges as a worldwide pandemic emerged. The COVID-19 pandemic has caused millions of people to be infected and die. The pandemic has also deteriorated population mental health. Specifically, the risks of morbidity and mortality associated with COVID-19 raised the fear about one's own health and the health of their families. Besides, the economic turmoil caused by the pandemic pushed many individuals to the brink of joblessness, which could be psychologically devastating to them $[1,2]$. In the USA, it is documented that as the pandemic severity proxied by the mortality rate is negatively associated with public mental health [3]. Compared to the Chinese, Americans tend to report more stress and depressive symptoms, which could potentially be attributed to their high number of infected healthcare workers, the lack of personal protective equipment, and the risk of losing health insurance tied to their employment [4].

In order to resolve the COVID-19 pandemic, attempts to produce vaccines have been made since early 2020 and the US Congress set aside billions of dollars for this purpose [5]. As a result, two series of vaccines, the Pfizer-BioNTech
COVID-19 vaccine and the Moderna COVID-19 vaccine, were successfully developed and granted emergency use authorization in December 2020. Mass vaccination in the USA immediately began in late 2020 .

This study evaluates the extent to which COVID-19 vaccination affects public mental health in the context of the USA. The study makes two contributions to the literature. First, I analyze the less tangible impacts of COVID-19 vaccination, particularly, the impact on mental health, whereas other studies tend to concentrate on the visible impacts such as those on infection, hospitalization, and death. By doing so, this study provides additional scientific evidence on the relationship between pandemic-related interventions and public welfare. Second, I attempt to overcome the endogeneity issue in prior studies on the topic of COVID-19. Specifically, the empirical model exploits the within-state and within-survey week variation in the number of people fully vaccinated per 10 million people.

I find that COVID-19 vaccination improves public mental health. Specifically, individuals are $0.24,0.23,0.12$, and 0.14 percentage points less likely to feel anxious, worried, displeased, and depressed every day, in response to a $100 \%$ increase in the number fully vaccinated per 10 million people. They are also 0.27 percentage points less likely to experience one of the four above-mentioned symptoms on a 
daily basis. These estimates correspond to the 1.46, 2.07, $1.40,1.61$, and $1.33 \%$ declines relative to the sample averages.

The study emphasizes the less discernible benefit of a pharmaceutical intervention, COVID-19 vaccination, on the mental well-being of individuals. The findings suggest that vaccine coverage expansion is imperative, especially for disproportionately affected communities. To the extent that the success of vaccines not only depends on production and availability but also on the uptake, it is crucial to ensure public confidence through effective communication on the benefits of vaccines. Since the COVID-19 vaccine is not sufficient to put an end to the pandemic, it is necessary to combine with other nonpharmaceutical interventions to effectively contain the outbreak and protect public wellbeing [6].

The paper proceeds as follows. Section 2 summarizes related literature. Section 3 describes the data. Section 4 provides the empirical model. Section 5 discusses the results. Section 6 concludes.

\section{Literature Review}

This study is related to two strands of literature. The first strand of literature focuses on government policies in response to the COVID-19 pandemic. One policy is the requirement of mask-wearing in public spaces. There is evidence that such a public mask mandate can reduce COVID-19-related symptoms, infection, and mortality $[7,8]$. Besides, the government can implement the massive lockdown that requires people to stay at home and businesses to shut down. It is reported that massive lockdown is effective at curtailing virus transmission, thus reducing COVID-19 infection and COVID-19-related deaths $[9,10]$. However, lockdown entails nonnegligible economic costs such as employment loss [11] and the depreciation of individual mental well-being [12-14]. Another policy response is the eviction moratorium that temporarily halts evictions and suspends foreclosures. The eviction moratorium allows extension on rent payments for financially distressed tenants due to COVID-19. Such a policy is also effective at reducing COVID-19 infection and mortality rates [15]. Moreover, expansionary monetary and fiscal policies are regarded as important tools to mitigate the economic downturn caused by the COVID-19 pandemic [16, 17]. While those studies concentrate on the nonpharmaceutical measures to contain the adverse consequences of the pandemic, this study contributes to the literature by analyzing the effects of a pharmaceutical intervention, namely the administering of COVID-19 vaccine doses on the population.

The second line of literature this study also fits into is the branch of studies on the impacts of COVID-19 vaccines. It is documented that vaccination can lessen the severity of the pandemic by downsizing COVID-19 infection, hospitalization, and death. Particularly, conducting a retrospective cohort study of consecutive, asymptomatic adult patients in the USA, Tande et al. [18] show that COVID-19 vaccination is associated with reduced asymptomatic infections. Studying the UK COVID-19 vaccine delivery plan, Cook and Roberts [19] find that vaccination decreases COVID-19 deaths, hospitalizations, and ICU admissions, with more rapid impacts on COVID-19 deaths. The authors also show that the impact of vaccination on hospitalization and ICU admissions can be further increased by the early prioritization of healthcare staff and clinically vulnerable patients. Besides, there is also evidence for the impacts of COVID-19 vaccination on the economy. Arnon and Ricco [20] report that doubling the number of vaccine doses administered daily might boost employment by more than 2 million and real GDP by about $1 \%$. I contribute to this line of literature by evaluating the less tangible impacts of COVID-19 vaccination, that is, the impacts on population mental health. Therefore, this study sheds additional light on the relationship between pharmaceutical interventions during the pandemic, the COVID-19 vaccination, on population wellbeing.

\section{Data}

3.1. Mental Health Data. Data on mental health are retrieved from the Household Pulse Survey Public Use File (HPSPUF). Conducted by the United States Census Bureau in cooperation with other US government agencies such as Bureau of Labor Statistics, the National Center for Health Statistics, the United States Department of Agriculture's Economic Research Service, the National Center for Education Statistics, and the Department of Housing and Urban Development, the HPS-PUF is a dataset that provides a good picture of American experiences during the COVID-19 pandemic. Besides rich information on mental health, the HPS-PUF also provides other characteristics of respondents such as race, age, marital status, education, and state of residence, among others.

There have been four phases of data collection so far. Phase 1 began on April 23, 2020, and ended on July 21, 2020. Phase 2 began on August 19, 2020, and ended on October 26, 2020. Phase 3 began on October 28, 2020, and ended on March 29, 2021. Phase 3.1 has just begun on April 14, 2021, and the first release was made on May 5, 2021. Despite the weekly collection and dissemination of data in phase 1 , the two-week collection and dissemination approach has been implemented since phase 2. It is worth noting that the collection periods are still called "weeks" in the HPS-PUF to maintain continuity [21]. In total, there are currently 30 survey weeks (waves) available, all of which are employed in this study.

Measures of individual mental health are based on responses to the questions about the frequency of displaying the following four psychological symptoms over the previous 7 days, including (i) the frequency of feeling nervous, anxious, or on edge, (ii) the frequency of not being able to stop or control worrying, (iii) the frequency of having little interest or pleasure in doing things, and (iv) the frequency of feeling down, depressed, or hopeless. Responses are put into a four-point scale as follows: 1: not at all, 2: several days, 3: more than half the days, and 4: nearly every day. Based on these responses, I construct four measures of mental health, namely daily anxiety, daily worry, daily displeasure, and daily depression. They are indicator variables taking the 
value of one if the respondent chooses the worst state (4: nearly every day), zero otherwise.

In addition to these four indicators, I construct another variable, psychological distress, to capture individual mental well-being. Psychological distress takes the value of one if the respondent exhibits at least one of the four symptoms (anxiety, worry, displeasure, and depression) on a daily basis, zero otherwise. In other words, psychological distress equals one if at least one variable out of the four variables, daily anxiety, daily worry, daily displeasure, and daily depression, takes the value of one, zero otherwise.

3.2. Data on COVID-19 Vaccination. The COVID-19 vaccination data are drawn from the "COVID-19 Vaccinations in the United States Jurisdiction" collection published by the Centers for Disease Control and Prevention (CDC). I obtain the number of fully vaccinated people at the week level for each state. Fully vaccinated people are those who received complete doses of a vaccine series (two doses of vaccines). The weekly interval is chosen because the HPS-PUF is collected and disseminated on a weekly basis. The lowest residential level in both the HPS-PUF and the vaccination data is the state.

My measure of COVID-19 vaccination is the number of people who received complete doses of a vaccine series in a week from the survey start date, then divided by 10,000,000. For example, consider an individual living in New York state and being surveyed in week 26 (March 3-March 15, 2021). The COVID-19 vaccination measure that the individual was exposed to is calculated as the number of new people in New York who have received complete doses of a vaccine series a week from the survey start date, that is, from February 17 to March 3, 2021, divided by 10,000,000 (week here refers to the data collection period, i.e., the survey week. Collection periods are still called "week" in the HPS-PUF despite the two-week collection and dissemination approach since phase 2). The main explanatory variable, log series complete, is the log of one plus the COVID-19 vaccination measure described above. The addition of one is intended to deal with zero number of fully vaccinated people, especially at the beginning of the pandemic.

3.3. Descriptive Statistics. The sample consists of over 2 million individuals. Descriptive statistics of the dependent and explanatory variables are displayed in panels $\mathrm{A}$ and $\mathrm{B}$ of Table 1, respectively. Evident from panel A, approximately $16.43,11.19,8.66$, and $8.79 \%$ of individuals report feeling anxious, worried, displeased, and depressed on a daily basis. Around $20.37 \%$ have at least one of these four feelings daily. As shown in panel $\mathrm{B}$, the average number of people who received complete doses of a vaccine series per 10 million people across the state is $2.96 \%$. The number is low because the period of the data starts from the beginning of the pandemic (week 1: April 23-May 5) when the vaccine was not available. The mean value of the main explanatory variable, log series complete, is 1.65 . The proportions of white, black, and other-race individuals are 82.4, 7.8, and 9.6\%, respectively. Roughly $57.7 \%$ are married, and $40.5 \%$ are male.
TABLe 1: Summary statistics.

\begin{tabular}{lccc}
\hline & $\begin{array}{c}\text { Mean } \\
(1)\end{array}$ & $\begin{array}{c}\text { SD } \\
(2)\end{array}$ & $\begin{array}{c}\text { Observations } \\
(3)\end{array}$ \\
\hline $\begin{array}{l}\text { Panel A: dependent variables } \\
\text { Daily anxiety }\end{array}$ & 0.1643 & 0.3705 & 2217646 \\
Daily worry & 0.1119 & 0.3153 & 2215454 \\
Daily displeasure & 0.0866 & 0.2812 & 2214180 \\
Daily depression & 0.0879 & 0.2832 & 2215895 \\
Psychological distress & 0.2037 & 0.4028 & 2208948 \\
\hline Panel B: independent variables & & & \\
Log series complete & 1.6548 & 4.5646 & 2543614 \\
Series complete per & 0.0296 & 0.1300 & 2543614 \\
10 million people & 0.8243 & 0.3806 & 2543614 \\
Respondent is white & 0.0789 & 0.2696 & 2543614 \\
Respondent is black & 0.0968 & 0.2956 & 2543614 \\
Neither white nor black & 51.812 & 15.833 & 2543614 \\
Age of respondent & 0.5774 & 0.4940 & 2543614 \\
Respondent is married & 0.4053 & 0.4909 & 2543614 \\
Respondent is male & & & \\
Respondent has bachelor's & 0.8624 & 0.3445 & 2543614 \\
degree & & & \\
Respondent works for & 0.1003 & 0.3004 & 2543614 \\
government & & & \\
\hline
\end{tabular}

The fraction of individuals having bachelor's degrees is $86.2 \%$, and the fraction working for the government is $10 \%$.

\section{Empirical Methodology}

To quantify the relationship between COVID-19 vaccination and mental health, I estimate the following regression equation:

$$
Y_{i s w}=\beta_{0}+\beta_{1} L S C_{s w}+\delta_{s}+\theta_{w}+X_{i s w}^{\prime} \Omega+\epsilon_{i s w},
$$

where the subscripts $i, s$, and $w$ correspond to individual, state of residence, and week of the survey, respectively. $Y_{i s w}$ represents the outcome variables, including daily anxiety (whether the individual feels anxious every day in the past seven days), daily worry (whether the individual feels worried on a daily basis in the past seven days), daily displeasure (whether the individual has the feeling of displeasure every day in the past seven days), daily depression (whether the individual feels depressed on a daily basis in the past seven days), and psychological distress (whether the individual has at least one among the above-mentioned four feelings daily in the past seven days).

The main explanatory variable, $L S C_{s w}$ (log series complete), is the log one plus the weekly number of people receiving two doses of a vaccine series per 10 million people for each state. The weekly COVID-19 vaccination measure is calculated by dividing the number of new people who have received complete doses of a vaccine series during the week preceding the survey date in the individual's state of residence by $10,000,000$. I denote by $\delta_{s}$ and $\theta_{w}$ the state and survey week fixed effects, respectively. Vector $X_{i s w}{ }^{\prime}$ is a covariate of individual characteristics including race, age, marital status, gender, education, and occupational category. Finally, $\epsilon_{i s w}$ is the error term. Standard errors throughout the paper are clustered at the state-by-week level. 
The coefficient of interest is $\beta_{1}$ that captures the effects of COVID-19 vaccination on mental health. To put it differently, $\beta_{1}$ reflects the change in the individual's psychological well-being as more people in his/her state of residence become fully vaccinated. Within this empirical setup, the identification comes from the variation in the number fully vaccinated per 10 million people on a weekly basis within the same state. The variation is due to the timing of the survey if it is within the state.

\section{Results}

\subsection{Main Results}

5.1.1. Daily Anxiety. The estimated impacts of COVID-19 vaccination on the daily feeling of anxiety are displayed in Table 2. In the most parsimonious specification that only conditions on the main explanatory variable (log series complete), I find that a $100 \%$ increase in the number fully vaccinated per 10 million people is linked with a 0.28 percentage point decrease in the probability of the individual feeling anxious every day (column 1). Without adequate controls for factors jointly influencing individual mental health and COVID-19 vaccination, the estimate only represents a correlation between vaccination and the daily feeling of anxiety.

In column 2, I include state fixed effects and survey week fixed effects to account for unobserved factors that could be correlated with vaccination and individual mental health. I find that individuals are 0.27 percentage points less likely to feel anxious on a daily basis in response to a $100 \%$ increase in the number receiving complete doses of a vaccine series per 10 million people.

Finally, in the most extensive specification that controls for individual characteristics (age, age-squared, marital status, race, gender, occupational sector, and educational attainment) in addition to the state and week fixed effects, I find negative and statistically significant impacts of COVID19 vaccination on the incidence of anxiety every day (column 3). Specifically, a $100 \%$ increase in the weekly number fully vaccinated per 10 million people in their state of residence makes individuals 0.24 percentage points less likely to experience anxiety on a daily basis. Taking the fraction of individuals reporting daily anxiety as a benchmark, the estimate represents a decrease of $1.46 \%$. Overall, Table 2 suggests that vaccination improves individual feelings of anxiety every day.

5.1.2. Daily Worry. The estimates for the relationship between COVID-19 vaccination and the incidence of daily worry are reported in Table 3. Evident from column 1, as the number fully vaccinated per 10 million people rise by $100 \%$, individuals are 0.18 percentage points less likely to feel worried on a daily basis. With the inclusion of state and survey week fixed effects, the incidence of daily worry reduces by 0.26 percentage points in response to a $100 \%$ increase in the weekly number receiving complete doses of a vaccine series per 10 million people in the state of residence (column 2).
TABLE 2: Vaccination and anxiety.

\begin{tabular}{lccc}
\hline & \multicolumn{3}{c}{$Y=$ daily anxiety } \\
& $(1)$ & $(2)$ & $(3)$ \\
\hline Log series complete & $-0.0028^{* * *}$ & $-0.0027^{* * *}$ & $-0.0024^{* * *}$ \\
Observations & $(0.0001)$ & $(0.0007)$ & $(0.0007)$ \\
All characteristics & 2217646 & 2217646 & 2217646 \\
All fixed effects & & & $\mathrm{X}$ \\
\hline
\end{tabular}

Note: ${ }^{*} p<0.1,{ }^{* *} p<0.05$, and ${ }^{* * *} p<0.01$. Daily anxiety is an indicator taking the value of one if the individual feels nervous, anxious, or on edge every day, zero otherwise. All characteristics include age, age-squared, marital status, race, gender, occupational sector, and educational attainment. All fixed effects include state and week fixed effects. Robust standard errors are clustered at the state-by-week level.

TABLE 3: Vaccination and worry.

\begin{tabular}{lccc}
\hline & \multicolumn{3}{c}{$Y=$ daily worry } \\
& $(1)$ & $(2)$ & $(3)$ \\
\hline Log series complete & $-0.0018^{* * *}$ & $-0.0026^{* * *}$ & $-0.0023^{* * *}$ \\
Observations & $(0.0000)$ & $(0.0006)$ & $(0.0006)$ \\
All characteristics & 2215454 & 2215454 & 2215454 \\
All fixed effects & & & $\mathrm{X}$ \\
\hline
\end{tabular}

Note: ${ }^{*} p<0.1,{ }^{* *} p<0.05$, and ${ }^{* * *} p<0.01$. Daily worry is an indicator taking the value of one if the individual cannot stop or control worry every day, zero otherwise. All characteristics include age, age-squared, marital status, race, gender, occupational sector, and educational attainment. All fixed effects include state and week fixed effects. Robust standard errors are clustered at the state-by-week level.

In the most extensive specification that controls for individual characteristics, state, and survey week fixed effects, I find that doubling the number fully vaccinated per 10 million people decreases the likelihood of individuals feeling worried on a daily basis by 0.24 percentage points. The estimate corresponds to a $2.07 \%$ reduction compared to the sample average. Taken together, Table 3 suggests that COVID-19 vaccination improves individual mental health by decreasing the daily feeling of worry.

5.1.3. Daily Displeasure. The estimated impacts of COVID19 vaccination and the incidence of daily displeasure are provided in Table 4. The most parsimonious specification shows a negative correlation between the COVID-19 vaccination and the probability of an individual feeling displeased every day (column 1). Controlling for unobserved factors influencing the number fully vaccinated and individual mental health through the state and survey week fixed effects, I find that doubling the weekly additional number receiving complete doses of a vaccine series per 10 million people in their state of residence makes individuals 0.15 percentage points less likely to feel displeased every day (column 2).

With a full set of individual controls and fixed effects, I find that a $100 \%$ increase in the number fully vaccinated per 10 million people is associated with a 0.12 percentage point decrease in the incidence of daily displeasure. Taking the 
TABLE 4: Vaccination and displeasure.

\begin{tabular}{lccc}
\hline & \multicolumn{3}{c}{$Y=$ daily displeasure } \\
& $(1)$ & $(2)$ & $(3)$ \\
\hline Log series complete & $-0.0008^{* * *}$ & $-0.0015^{* * *}$ & $-0.0012^{* *}$ \\
Observations & $(0.0000)$ & $(0.0006)$ & $(0.0006)$ \\
All characteristics & 2214180 & 2214180 & 2214180 \\
All fixed effects & & & $\mathrm{X}$ \\
\hline
\end{tabular}

Note: ${ }^{*} p<0.1,{ }^{* *} p<0.05$, and ${ }^{* * *} p<0.01$. Daily displeasure is an indicator taking the value of one if the individual has little interest or pleasure in doing things every day, zero otherwise. All characteristics include age, agesquared, marital status, race, gender, occupational sector, and educational attainment. All fixed effects include state and week fixed effects. Robust standard errors are clustered at the state-by-week level.

proportion of individuals feeling displeased every day as the benchmark, the estimate implies a reduction of $1.40 \%$. Table 4 suggests that COVID-19 vaccination enhances individual psychological well-being by decreasing the daily feeling of displeasure.

5.1.4. Daily Depression. I show the relationship between COVID-19 vaccination and the feeling of daily depression in Table 5. According to the most parsimonious specification that only controls for the log weekly number of fully vaccinated per 10 million people, I detect a negative association between COVID-19 vaccination and the probability of an individual feeling depressed on a daily basis (column 1). With the inclusion of state and survey week fixed effects, the estimate increases slightly in magnitude, and the statistical significance level is preserved (column 2). Particularly, a $100 \%$ increase in the number receiving complete doses of a vaccine series per 10 million people in the week preceding the survey week in the individual's state of residence makes him/her 0.16 percentage points less likely to be depressed daily.

Finally, the most extensive specification points to a 0.14 percentage point reduction in the incidence of daily depression in response to a $100 \%$ increase in the number fully vaccinated per 10 million people, which corresponds to the $1.61 \%$ decline relative to the sample average. Collectively, Table 5 suggests that COVID-19 vaccination improves individual mental well-being by decreasing the daily feeling of depression.

5.1.5. Psychological Distress. The estimated impacts of COVID-19 vaccination on the probability of an individual having at least one of the four symptoms (anxiety, worry, displeasure, and depression) on a daily basis are displayed in Table 6 . The outcome variable in the table is psychological distress that takes the value of one if the individual reports at least one feeling among the above-mentioned four every day, zero otherwise. Psychological distress can capture individual overall mental well-being because having one of these four symptoms is enough to be considered psychologically affected.

Without controlling for state and survey fixed effects as well as individual characteristics, I find that a $100 \%$ increase
TABLE 5: Vaccination and depression.

\begin{tabular}{lccc}
\hline & \multicolumn{3}{c}{$Y=$ daily depression } \\
& $(1)$ & $(2)$ & $(3)$ \\
\hline Log series complete & $-0.0010^{* * *}$ & $-0.0016^{* * *}$ & $-0.0014^{* *}$ \\
Observations & $(0.0000)$ & $(0.0006)$ & $(0.0006)$ \\
All characteristics & 2215895 & 2215895 & 2215895 \\
All fixed effects & & & $\mathrm{X}$ \\
\hline
\end{tabular}

Note: ${ }^{*} p<0.1,{ }^{* *} p<0.05$, and ${ }^{* * *} p<0.01$. Daily depression is an indicator taking the value of one if the individual feels down, depressed, or hopeless every day, zero otherwise. All characteristics include age, age-squared, marital status, race, gender, occupational sector, and educational attainment. All fixed effects include state and week fixed effects. Robust standard errors are clustered at the state-by-week level.

in the weekly number fully vaccinated per 10 million people is linked to a 0.32 percentage point decrease in the probability of an individual feeling psychologically distressed every day (column 1). Accounting for state and survey fixed effects leaves the estimate virtually unchanged (column 2). According to the most extensive specification in column 3, as more people get fully vaccinated, individual mental health improves. Specifically, doubling the number receiving complete doses of a vaccine series per 10 million people makes individuals 0.27 percentage points less likely to have at least one of the four psychologically negative experiences (anxiety, worry, displeasure, and depression) every day. The estimate corresponds to a $1.33 \%$ decline in the incidence of psychological distress. Overall, Table 6 suggests that COVID-19 vaccination enhances individual mental health.

5.2. Other Measures. So far I have provided evidence that COVID-19 vaccination can improve individuals' mental health. In this section, I adopt different measures of the explanatory variable and outcome variables to test for the robustness of the results. The estimating results are displayed in Tables 7 and 8.

5.2.1. Other Explanatory Measures. First, I adopt different measures of the main explanatory variable. Recall that in the previous section, the explanatory variable, log series complete, is the log of one plus the number fully vaccinated per 10 million people in the week preceding the survey week in the individual's state of residence. Now I replace the log measure with the nominal measure, series complete per 10 million people, which is the number receiving complete doses of a vaccine series per 10 million people as described above. According to panel A of Table 7, as more people get fully vaccinated in their state of residence, individuals' mental health improves. Estimates are negative and statistically significant. Second, I replace the log measure with the standardized measure of the number receiving complete doses of a vaccine series per 10 million people as the main explanatory variable. Doing so does not change my conclusion as panel B of Table 7 still points to the negative relationship between vaccination and psychological burden. Taken together, the results are robust to the utilization of different explanatory variables. 
TABLE 6: Vaccination and psychological distress.

\begin{tabular}{lccc}
\hline & & $Y=$ psychological distress & $(2)$ \\
& $(1)$ & $-0.0032^{* * *}$ & $-0.0027^{* * *}$ \\
Log series complete & $-0.0032^{* * *}$ & $(0.0008)$ & $(0.0008)$ \\
Observations & $(0.0001)$ & 2208948 & 2208948 \\
All characteristics & 2208948 & & $\mathrm{X}$ \\
All fixed effects & & $\mathrm{X}$ & $\mathrm{X}$ \\
\hline
\end{tabular}

Note: ${ }^{*} p<0.1,{ }^{* *} p<0.05$, and ${ }^{* * *} p<0.01$. Psychological distress is an indicator taking the value of one if the individual has at least one of the following feelings on a daily basis, namely, anxiety, worry, displeasure, and depression, zero otherwise. All characteristics include age, age-squared, marital status, race, gender, occupational sector, and educational attainment. All fixed effects include state and week fixed effects. Robust standard errors are clustered at the state-by-week level.

TABLE 7: Vaccination and mental health-other explanatory measures.

\begin{tabular}{|c|c|c|c|c|c|}
\hline & $\begin{array}{l}\text { Daily anxiety } \\
(1)\end{array}$ & $\begin{array}{l}\text { Daily worry } \\
\text { (2) }\end{array}$ & $\begin{array}{c}\text { Daily displeasure } \\
\text { (3) }\end{array}$ & $\begin{array}{c}\text { Daily depression } \\
(4)\end{array}$ & $\begin{array}{c}\text { Psychological distress } \\
\text { (5) }\end{array}$ \\
\hline \multicolumn{6}{|l|}{ Panel A: nominal measure } \\
\hline Series complete per 10 million people & $\begin{array}{c}-0.0624^{* * *} \\
(0.0020)\end{array}$ & $\begin{array}{c}-0.0370^{* * *} \\
(0.0017)\end{array}$ & $\begin{array}{c}-0.0165^{* * *} \\
(0.0015)\end{array}$ & $\begin{array}{c}-0.0206^{* * *} \\
(0.0015)\end{array}$ & $\begin{array}{c}-0.0698^{* * *} \\
(0.0021)\end{array}$ \\
\hline $\begin{array}{l}\text { Observations } \\
\text { Panel B: standardized measure }\end{array}$ & $2,217,646$ & $2,215,454$ & $2,214,180$ & $2,215,895$ & $2,208,948$ \\
\hline Standardized series complete & $\begin{array}{c}-0.0081^{* * *} \\
(0.056)\end{array}$ & $\begin{array}{c}-0.0048^{* * *} \\
(0.053)\end{array}$ & $\begin{array}{c}-0.0021^{* * *} \\
(0.053)\end{array}$ & $\begin{array}{c}-0.0027^{* * *} \\
(0.053)\end{array}$ & $\begin{array}{c}-0.0091^{* * *} \\
(0.053)\end{array}$ \\
\hline Observations & $2,217,646$ & $2,215,454$ & $2,214,180$ & $2,215,895$ & $2,208,948$ \\
\hline All characteristics & $\mathrm{X}$ & $\mathrm{X}$ & $\mathrm{X}$ & $\mathrm{X}$ & $\mathrm{X}$ \\
\hline All fixed effects & $\mathrm{X}$ & $\mathrm{X}$ & $\mathrm{X}$ & $\mathrm{X}$ & $\mathrm{X}$ \\
\hline
\end{tabular}

Note: ${ }^{*} p<0.1,{ }^{* *} p<0.05$, and ${ }^{* * *} p<0.01$. All characteristics include age, age-squared, marital status, race, gender, occupational sector, and educational attainment. All fixed effects include state and week fixed effects. Robust standard errors are clustered at the state-by-week level.

TABLE 8: Vaccination and mental health-other outcome measures.

\begin{tabular}{lccccc}
\hline & Uncoded anxiety & Uncoded worry & Uncoded displeasure & Uncoded depression & Psychological distress (avg.) \\
& $(1)$ & $(2)$ & $(2)$ & $(2)$ & $-0.0044^{* *}$ \\
Log series complete & $-0.0041^{* *}$ & $-0.0057^{* * *}$ & $-0.0058^{* * *}$ & $(0.0018)$ & $\left(0.00179^{* * *}\right.$ \\
Observations & $(0.0021)$ & $(0.0020)$ & $(0.0019)$ & 2215895 & 2220666 \\
All characteristics & 2217646 & 2215454 & 2214180 & $\mathrm{X}$ & $\mathrm{X}$ \\
All fixed effects & $\mathrm{X}$ & $\mathrm{X}$ & $\mathrm{X}$ & $\mathrm{X}$ & $\mathrm{X}$ \\
\hline
\end{tabular}

Note: ${ }^{*} p<0.1,{ }^{* *} p<0.05$, and ${ }^{* * *} p<0.01$. All characteristics include age, age-squared, marital status, race, gender, occupational sector, and educational attainment. All fixed effects include state and week fixed effects. Robust standard errors are clustered at the state-by-week level.

5.2.2. Other Outcome Measures. I proceed to employ different measures of mental health. Recall that in Section 5.1, dependent variables are daily anxiety, daily worry, daily displeasure, and daily depression, which take the value of one if the individual reports feeling anxious, worried, displeased, and depressed on a daily basis, respectively, zero otherwise. Psychological distress is a dummy variable taking the value of one if at least one of the above-mentioned outcome variables equals one, zero otherwise.

In this section, I utilize the uncoded measures of mental health, uncoded anxiety, uncoded worry, uncoded displeasure, and uncoded depression, which refers to the frequency of experiencing each feeling. Frequency is expressed in a four-point scale as follows: 1: not at all, 2: several days, 3: more than half the days, and 4: nearly every day. To capture the overall mental health condition, I construct the variable psychological distress (average) that is calculated as the average of the uncoded anxiety, uncoded worry, uncoded displeasure, and uncoded depression variables. Table 8 shows that adopting uncoded measures of mental health leaves my conclusion on the relationship between COVID19 vaccination and individual psychological well-being unchanged.

5.3. Discussion. Collectively, I have presented evidence that COVID-19 vaccination improves individual mental health. Specifically, a $100 \%$ increase in the number fully vaccinated per 10 million people makes individuals $0.24,0.23,0.12$, and 0.14 percentage points less likely to feel anxious, worried, displeased, and depressed on a daily basis, respectively. The probability of having at least one of the four symptoms (anxiety, worry, displeasure, and depression) every day also declines by 0.27 percentage points. Taking the fractions of individuals reporting such psychological problems as the benchmark, these estimates imply the corresponding 
declines of $1.46,2.07,1.40,1.61$, and $1.33 \%$. The findings are insensitive to the utilization of different measures of outcome and explanatory variables.

These findings are consistent with prior studies on the positive impacts of COVID-19 vaccination. In particular, vaccination has been shown to decrease COVID-19 infection, deaths, and hospital admissions [18, 19]. In other words, such a pharmaceutical intervention is effective at combating the COVID-19 pandemic. Furthermore, COVID-19 vaccination is also documented to favorably affect the economy as shown in Arnon and Ricco [20]. Specifically, the authors find that doubling the number of vaccine doses administered daily might boost employment by more than 2 million and real GDP by approximately $1 \%$. By presenting evidence that COVID-19 vaccination improves population mental health, this study contributes to the line of studies on the role of vaccines in averting the disruptions imposed by the pandemic.

There could be multiple potential mechanisms through which COVID-19 vaccination positively affects individual psychological well-being. First, as more people got vaccinated; others will be better protected from being infected with COVID-19, especially those of high risk for severe illness from the virus such as the elderly and those with medical conditions [22]. The sense of protection due to high levels of vaccination may improve individuals' psychological well-being. Also related to this point, it is documented that the more severe the COVID-19 pandemic gets, the heavier the toll it takes on individual mental health [3]. Therefore, by lessening the severity of the pandemic through downsizing infection, curtailing hospitalization, and reducing deaths $[18,19]$, COVID-19 vaccination could improve individual mental health. Second, as an important tool to control the pandemic, vaccination might lead to the relaxation of various stringent nonpharmaceutical measures such as lockdown that has been shown to adversely affect psychological well-being $[12,14]$. As a result, population mental health can be enhanced in response to a higher level of COVID-19 vaccination. Finally, besides creating a health emergency, the COVID-19 pandemic also generated enormous economic turmoil, pushing millions of people into unemployment $[1,2]$. As vaccination can bring about economic recovery [20], the resulting rise in employment may alleviate individuals' financial strain, thus improving their psychological well-being.

This study highlights the less visible benefit of a pharmaceutical intervention, COVID-19 vaccination, on the mental well-being of individuals. In other words, COVID-19 vaccination can curtail the psychological burden on the population that was generated by the pandemic. Since poor mental health can disrupt everyday life, incite antisocial behaviors, and elevate the risk of cardiovascular diseases [23-25], by improving individuals' mental health, COVID19 vaccination might have meaningful implications in the long run. Therefore, this study underlines the importance of COVID-19 vaccination to public well-being. It is imperative to expand vaccine coverage, especially for disproportionately affected communities and developing countries. To the extent that the success of vaccines not only depends on production and availability but also on the uptake, raising confidence in the public healthcare system and in recommendations from the government through effective communication is important to increase support for the COVID19 vaccine as well as to expand vaccine coverage [26]. Indeed, the authors show the overall high acceptance rate of the COVID-19 vaccine and the higher willingness to pay for vaccines among psychiatric patients in a context where public confidence in the benefits of vaccines is ensured. Besides, since the COVID-19 vaccine is not a panacea, it is necessary to combine with other nonpharmaceutical interventions to effectively contain the outbreak and protect public well-being [6].

While this study highlights the positive impacts of COVID-19 vaccination on population mental health, it is worth noting that besides vaccines, there are other determinants of mental health. Physical health, access to information on the pandemic, and local government responses are important factors. Specifically, as for physical health, Wang et al. [4] show that individuals experiencing COVID19-related symptoms tend to have their mental health aggravated. Regarding access to information on the pandemic, increased Internet use for information-searching purposes might lead to higher depression symptoms [27]. Being aware of the mortality statistics is associated with worse psychological well-being [3]. Nevertheless, knowledge of other countries' responses to the pandemic as well as the acquisition of reliable travel advice can positively affect mental health [4]. In terms of government responses, the provision of adequate health and prevention advice through effective communication between the local authority and the public can lessen the psychological burden of the pandemic [28].

\section{Conclusion}

This study contributes to the literature by evaluating the less tangible benefit of a pharmaceutical intervention during the COVID-19 pandemic, the administering of COVID-19 vaccine doses to the population, on public mental health. The data on mental health are retrieved from the Household Pulse Survey Public Use File (HPS-PUF), while the data on COVID-19 vaccination are obtained from the "COVID-19 Vaccinations in the United States Jurisdiction" collection published by the CDC. In terms of identification, the empirical model exploits the within-state and within-survey week variation in the number receiving complete doses of a vaccine series per 10 million people.

This study finds that COVID-19 vaccination positively affects individual mental health. Particularly, a 100\% increase in the number fully vaccinated per 10 million people makes individuals $0.24,0.23,0.12$, and 0.14 percentage points less likely to experience anxiety, worry, displeasure, and depression on a daily basis, respectively. The probability of having at least one of the four symptoms (anxiety, worry, displeasure, and depression) every day also reduces by 0.27 percentage points. These estimates correspond to the 1.46, $2.07,1.40,1.61$, and $1.33 \%$ declines relative to the sample averages. These findings on the relationship between COVID-19 vaccination and population mental health are 
insensitive to the utilization of different measures of explanatory and outcome variables.

These findings underscore the less discernible value of COVID-19 vaccination on public mental health. The study calls for the expansion of vaccine coverage, especially for disproportionately affected communities. Because the success of vaccines depends on not only production and availability but also on the uptake, it is crucial to ensure public confidence in the benefits of vaccines to curtail refusal and resistant attitudes toward vaccination. To the extent that vaccination is not sufficient to contain the pandemic, the combination with other nonpharmaceutical interventions is important to protect public mental well-being [6].

\section{Data Availability}

The data underlying this study can be obtained at https://www. census.gov/programs-surveys/household-pulse-survey/datasets. html and https://data.cdc.gov/Vaccinations/COVID-19-Vacc inations-in-the-United-States-Jurisdi/unsk-b7fc.

\section{Conflicts of Interest}

The author declares no conflicts of interest.

\section{Acknowledgments}

This study was supported by The Youth Incubator for Science and Technology Programe, managed by Youth Development Science and Technology Center-Ho Chi Minh Communist Youth Union and Department of Science and Technology of Ho Chi Minh City; the contract number is “10/2020/HĐ-KHCNT-VƯ ngày 30 tháng 12 năm 2020.”

\section{References}

[1] R. W. Fairlie, K. Couch, and H. Xu, "The impacts of COVID19 on minority unemployment: first evidence from April 2020 CPS microdata," W27246, National Bureau of Economic Research, Cambridge, MA, USA, 2020.

[2] F. L. Rojas, X. Jiang, L. Montenovo, K. I. Simon, B. A. Weinberg, and C. Wing, "Is the cure worse than the problem itself? immediate labor market effects of COVID-19 case rates and school closures in the US," W27127, National Bureau of Economic Research, Cambridge, MA, USA, 2020.

[3] K. Le and M. Nguyen, "The psychological burden of the COVID-19 pandemic severity," Economics and Human Biology, vol. 41, Article ID 100979, 2021a.

[4] C. Wang, C. Tripp, S. F. Sears et al., "The impact of the COVID-19 pandemic on physical and mental health in the two largest economies in the world: a comparison between the United States and China," Journal of Behavioral Medicine, pp. 1-19, 2021.

[5] The Small Business Administration and the Department of Treasury, The Small Business Owner's Guide to the CARES Act, The Small Business Administration and the Department of Treasury, Washington, DC, USA, 2020.

[6] S. Moore, E. M. Hill, M. J. Tildesley, L. Dyson, and M. J. Keeling, "Vaccination and non-pharmaceutical interventions for COVID-19: a mathematical modelling study," The Lancet Infectious Diseases, vol. 21, no. 6, pp. 793-802, 2021.
[7] W. Lyu and G. L. Wehby, "Community use of face masks and COVID-19: evidence from a natural experiment of state mandates in the US: study examines impact on COVID-19 growth rates associated with state government mandates requiring face mask use in public," Health Affairs, vol. 39, no. 8, pp. 1419-1425, 2020 b.

[8] M. Nguyen, "Mask mandates and COVID-19 related symptoms in the US," ClinicoEconomics and Outcomes Research: CEOR, vol. 13, p. 757, 2021a.

[9] J. Hellewell, S. Abbott, A. Gimma et al., "Feasibility of controlling COVID-19 outbreaks by isolation of cases and contacts," The Lancet Global Health, vol. 8, no. 4, 2020.

[10] J. Roux, C. Massonnaud, and P. Crepey, "COVID-19: onemonth impact of the french lockdown on the epidemic burden," medRxiv, 2020.

[11] O. Coibion, Y. Gorodnichenko, and M. Weber, "Labor markets during the COVID-19 crisis: a preliminary view," W27017, National Bureau of Economic Research, Cambridge, MA, USA, 2020.

[12] K. Le and M. Nguyen, "The psychological consequences of COVID-19 lockdowns," International Review of Applied Economics, vol. 35, no. 2, pp. 147-163, 2021b.

[13] M. Nguyen, "Internet access for children's online schooling during the COVID-19 pandemic and parental mental health," Review of International Geographical Education Online, vol. 11, no. 5, pp. 4909-4915, 2021 b.

[14] C. L. Niedzwiedz, M. J. Green, M. Benzeval et al., "Mental health and health behaviours before and during the initial phase of the COVID-19 lockdown: longitudinal analyses of the UK household longitudinal study," Journal of Epidemiology \& Community Health, vol. 75, no. 3, pp. 224-231, 2021.

[15] K. Jowers, C. Timmins, N. Bhavsar, Q. Hu, and J. Marshall, "Housing precarity \& the COVID-19 pandemic: impacts of utility disconnection and eviction moratoria on infections and deaths across US counties," w28394, National Bureau of Economic Research, Cambridge, MA, USA, 2021.

[16] F. Bianchi, R. Faccini, and L. Melosi, "Monetary and fiscal policies in times of large debt: unity is strength," w27112, National Bureau of Economic Research, Cambridge, MA, USA, 2020.

[17] P. O. Gourinchas, "Flattening the pandemic and recession curves," in Mitigating the COVID Economic Crisis: Act Fast and Do Whatever, R. Baldwin and B. W. d. Mauro, Eds., pp. 31-39, CEPR Press, London, UK, 2020.

[18] A. J. Tande, B. D. Pollock, N. D. Shah et al., "Impact of the COVID-19 vaccine on asymptomatic infection among patients undergoing pre-procedural COVID-19 molecular screening," Clinical Infectious Diseases, 2021.

[19] T. M. Cook and J. V. Roberts, "Impact of vaccination by priority group on UK deaths, hospital admissions and intensive care admissions from COVID-19," Anaesthesia, vol. 76, no. 5, pp. 608-616, 2021.

[20] A. Arnon and J. Ricco, "Epidemiological and economic effects of the COVID-19 vaccine in 2021: penn wharton budget model," 2021, https://budgetmodel.wharton.upenn.edu/ issues/2021/3/1/epidemiological-and-economic-effects-ofcovid-19-vaccine.

[21] United States Census Bureau, "Household pulse survey data tables," 2021, https:/www.census.gov/programs-surveys/ household-pulse-survey/data.html.

[22] Centers for Disease Control and Prevention (CDC), "Benefits of getting a COVID-19 vaccine,” 2021, https://www.cdc.gov/ coronavirus/2019-ncov/vaccines/vaccine-benefits.html. 
[23] A. K. Dhar and D. A. Barton, "Depression and the link with cardiovascular disease," Frontiers in Psychiatry, vol. 7, p. 33, 2016.

[24] D. Nutt, S. Wilson, and L. Paterson, "Sleep disorders as core symptoms of depression," Dialogues in Clinical Neuroscience, vol. 10, no. 3, p. 329, 2008.

[25] G. J. Rubin and S. Wessely, "The psychological effects of quarantining a city," BMJ, vol. 368, 2020.

[26] F. Hao, B. Wang, W. Tan et al., "Attitudes toward COVID-19 vaccination and willingness to pay: comparison of people with and without mental disorders in China," BJPsych Open, vol. 7, no. $5,2021$.

[27] D. Valdez, M. Ten Thij, K. Bathina, L. A. Rutter, and J. Bollen, "Social media insights into US mental health during the COVID-19 pandemic: longitudinal analysis of twitter data," Journal of Medical Internet Research, vol. 22, no. 12, Article ID e21418, 2020.

[28] B. Hyland-Wood, J. Gardner, J. Leask, and U. K. Ecker, "Toward effective government communication strategies in the era of COVID-19," Humanities and Social Sciences Communications, vol. 8, no. 1, pp. 1-11, 2021. 